12

\title{
Новый метод измерения показателя преломления текущей жидкости
}

\author{
() В.В. Давыдов, А.В. Мороз, Д.И. Николаев \\ Санкт-Петербургский политехнический университет Петра Великого, \\ 195251 Санкт-Петербург, Россия \\ e-mail: Davydov_vadim66@mail.ru
}

Поступила в редакцию 23.11.2020 г.

В окончательной редакции 07.02.2021 г.

Принята к публикации 18.02.2021 г.

\begin{abstract}
Обоснована необходимость разработки нового метода измерения показателя преломления жидкости при турбулентном режиме её течения в трубопроводе. Представлен новый метод измерения показателя преломления среды $n$. Для его практической реализации разработана новая конструкция проточного рефрактометра, позволяющая проводить измерения $n$ как при ламинарном, так и при турбулентном режиме течения жидкости. Рассмотрена новая конструкция оптической части рефрактометра. Предложен новый способ её размещения на вертикальном участке трубопровода для измерения $n$. Все это позволяет сделать несущественным влияние на результат измерения $n$ текущей жидкости погрешностей, связанных с многократными отражениями лазерного излучения от оптических элементов, с образованием пустот или вихревых потоков в трубопроводе и с колебаниями температуры. В конструкции рефрактометра с новым методом измерения $n$ отсутствуют ограничения по определению значения $n$ текущей жидкости, в отличие от используемых промышленных проточных рефрактометров, принцип работы которых основан на явлении полного внутреннего отражения лазерного излучения на границе двух сред. Представлены результаты экспериментальных исследований различных сред.
\end{abstract}

Ключевые слова: проточный рефрактометр, полное внутреннее отражение, мутность.

DOI: $10.21883 /$ OS.2021.07.51088.1660-21

\section{Введение}

В настоящее время в различных научных исследованиях, в системах охлаждения ядерных реакторов, токамаков и других устройств, в технологических процессах на производстве все чаще используют быстрые (турбулентные) потоки жидких сред [1-4]. В большинстве случаев поток формируется однофазной средой с высокой прозрачностью (вода, дистиллированная и тяжелая вода, спирт и т.д.) или двухфазной (различные соединения этиленгликоля, медицинские растворы, водные растворы щелочей и прочие) [2, 3,5-8].

Их состояние, а в ряде случаев и расход $q$ необходимо контролировать в диапазоне изменения скорости потока более чем на два порядка $[1,3,5,9-11]$. При этом температура $T$ текущей среды может изменяться более чем на $200 \mathrm{~K}$, например в энергетических установках и системах охлаждения токамаков. Необходимо также отметить, что проводимые измерения параметров текущей среды для определения её состояния не должны вносить необратимых изменений в её физическую структуру и химический состав [2-5,8-10,12-14]. Это необходимо, например, для обеспечения непрерывного процесса при проведении продолжительных научных экспериментов или при производстве различных жидких сред (в том числе опасных), например, в химической и фармацевтической промышленности. В последнем случае необходимо также обеспечить стерильность измерительного процесса.
В такой ситуации наиболее целесообразным решением является использование бесконтактных измерителей, например, ядерно-магнитных расходомеров - релаксометров $[2,3,5,8-10,15,16]$. Эти приборы позволяют одним и тем же устройством контролировать расход жидкости $q$ и состояние среды по изменению времен продольной $T_{1}$ и поперечной $T_{2}$ релаксации $[2,3,5,9,10,15,16]$. Однако в случае одновременного изменения температуры $T$ и расхода $q$ текущей среды в большом диапазоне (более чем на порядок) при эксплуатации ядерномагнитных расходомеров-релаксометров могут возникнуть сложно решаемые проблемы $[9,10,15]$. По этой причине для контроля расхода жидкости $q$ и её состояния используют два измерительных прибора: один для контроля расхода $q$, например электромагнитный или оптический расходомер, другой для контроля состояния среды, например проточный рефрактометр, принцип работы которого основан на явлении полного внутреннего отражения (ПВО) на границе двух сред [12,14,17-20]. Выпускаемые в настоящее время модели промышленных проточных рефрактометров, работающих на явлении ПВО при изменении расхода текущей среды более чем на два порядка с одновременным изменение её температуры $T$, обладают рядом недостатков. Одновременное изменение показателей преломления призмы в рефрактометре и текущей жидкости в трубопроводе при большом изменении температуры текущей среды создает ряд сложностей, особенно при реализации автоматической настройки оптической системы регистрации отражен- 


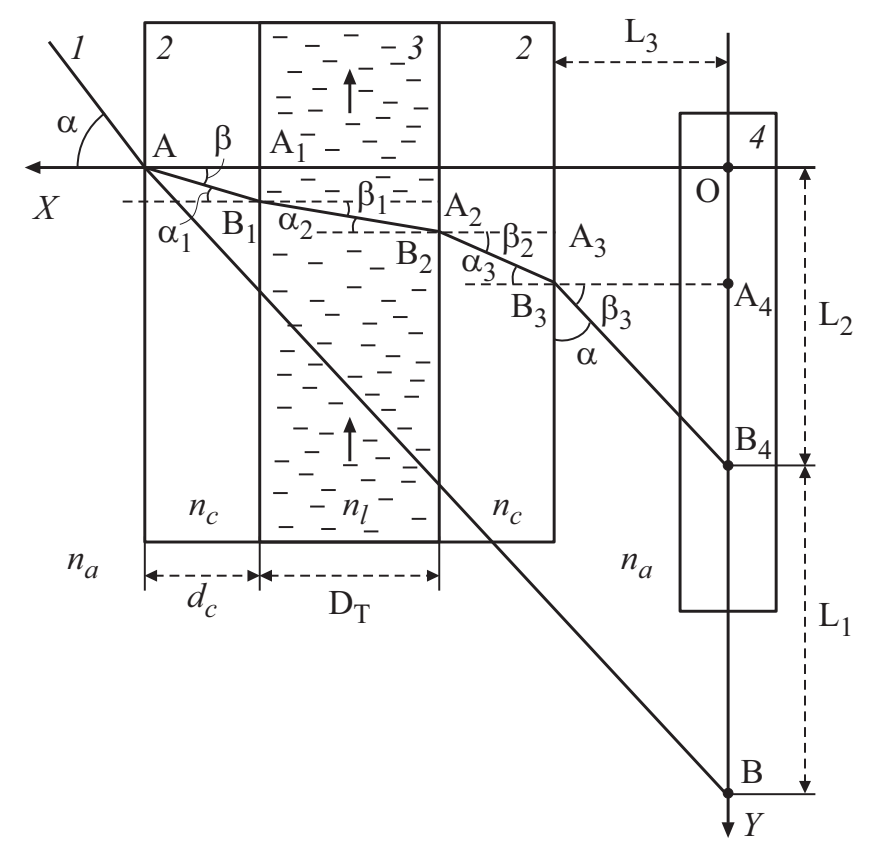

Рис. 1. Схема прохождения лазерного луча через стенки трубопровода, текущую жидкость и воздушное пространство до фотодиодной линейки: 1 - лазерный луч; 2 - стенка трубопровода; 3 - текущая жидкость; 4 - фотодиодная линейка.

ного лазерного излучения, что ограничивает диапазон измерения $n$. Кроме того, при больших скоростях потока жидкости явление сдвига Гуса-Хэнхена отраженного лазерного излучения приводит к увеличению погрешности измерения $n$.

Это не позволяет в ряде случаев проводить измерения показателя преломления $n$ текущей жидкости с погрешностью менее $10^{-4}$, которая необходима для достоверного контроля её состояния, особенно при проведении научных исследований. Поэтому разработка нового метода измерения показателя преломления текущих сред при больших скоростях потока при изменении их температур более чем на $200 \mathrm{~K}$ является актуальной задачей. Один из возможных вариантов её решения представлен в настоящей работе.

\section{1. Новый метод измерения показателя преломления текущей среды и конструкция рефрактометра для его реализации}

С учетом анализа различных методов измерения показателя преломления $n$ при исследовании состояния текущих сред [12,14,17-22] и конструкций промышленных проточных рефрактометров нами был разработан новый метод измерения показателя преломления текущей жидкости $n_{l}$.

Рассмотрим процесс прохождения лазерного излучения 1 через стенки трубопровода 2, текущую жидкость 3 и воздушный зазор (шириной $L_{3}$ ) между внешней стенкой трубопровода 2 и фотодиодной линейкой 4 (рис. 1). Рассматривается луч, соответствующий максимуму интенсивности в лазерном излучении. Для регистрации лазерного излучения используется фотодиодная линейка TSL1406RS, состоящая из 512 фоточувствительных сенсоров (компания AMS-TAOS, CША) с длиной фоточувствительного слоя $40.16 \mathrm{~mm}$. Размер фоточувствительного сенсора по оси $O Y$ составляет $0.74 \mathrm{~mm}$, по оси $O X-$ $1.2 \mathrm{~mm}$. Этого достаточно для регистрации максимума лазерного излучения на одном сенсоре. С использованием фотодиодной линейки 4 определяется положение максимума (координата точки $B_{4}-$ расстояние $L_{2}$ ). Измеряется на какое расстояние по оси $O Y$ сместился лазерный луч 1 относительно точки $A$ после прохождения всех сред.

В случае попадания максимума лазерного излучения в зазор между фоточувствительными сенсорами погрешность измерения расстояния $L_{2}$ после автоматической подстройки оптической системы будет не превышать величину $0.01 \mathrm{~mm}$. В конструкции фотодиодной линейки TSL1406RS перед фоточувствительным слоем нет фокусирующих оптических элементов, как в других моделях линеек. Поэтому влияние эффектов, связанных с неоднократным отражением лазерного излучения между фоточувствительным слоем и стенкой трубопровода, на отношение сигнал/шум в фоточувствительных сенсорах линейки будет несущественно.

Эффекты, связанные с поглощением лазерного излучения в текущей среде, при описании данного метода не рассматриваются, так как они оказывают влияние в первую очередь на величину интенсивности максимума лазерного излучения. Фоточувствительность сенсора линейки очень высокая. Это позволяет в достаточно большом диапазоне изменения прозрачности среды (увеличения мутности) четко определять наличие максимума в лазерном излучении. Далее экспериментально будет показано, как влияет увеличение поглощения лазерного излучения средой на погрешность измерения показателя преломления $n_{l}$.

Расстояние $L_{2}$ также можно вычислить, рассматривая преломление лазерного излучения на границах двух различных сред (воздух-оргстекло, оргстекло-текущая жидкость и текущая жидкость-оргстекло) (рис. 1). Для каждого случая преломления на границе двух сред вычисляется смещение луча относительно точки его падения на границу двух сред.

Рассмотрим для примера преломление на границе двух сред воздух-оргстекло (стенка трубопровода). Ход лучей представлен на рис. 1. Определим значение $\left|A_{1} B_{1}\right|$

$$
\begin{gathered}
\left|A_{1} B_{1}\right|=d_{c} \operatorname{tg} \beta, \\
\sin \alpha / \sin \beta=n_{c} / n_{a}, \\
\beta=\arcsin \left(\left(n_{a} \sin \alpha\right) / n_{c}\right), \\
\left|A_{1} B_{1}\right|=d_{c} \operatorname{tg}\left(\arcsin \left(\left(n_{a} \sin \alpha\right) / n_{c}\right),\right.
\end{gathered}
$$


где $n_{c}$ - показатель преломления оргстекла, $n_{a}-$ показатель преломления воздуха.

Подставлять в формулы (2)-(4) значение показателя преломления воздуха, равного $1.000295 \pm 0.000001$ (данное значение получено для атмосферного давления $101325 \mathrm{~Pa}$, влажности 50\%, температуре $20^{\circ} \mathrm{C}$ ) нецелесообразно, так как трубопровод может эксплуатироваться в различной воздушной среде, например насыщенной водяными парами, при более высоком давлении и температуре, отличающейся от $20^{\circ} \mathrm{C}$. В этом случае в значении па произойдут изменения в шестом или пятом знаке, а при больших изменениях влажности, давления и температуры - в четвертом.

Далее рассматривается преломление на границе двух сред оргстекло и текущая жидкость, а также оргстекло и воздух. Для этих случаев определим углы падения лазерного излучения на границы двух сред $\alpha_{1}, \alpha_{2}$ и $\alpha_{3}$ в точках $B_{1}, B_{2}$ и $B_{3}$, а также угол $\beta_{3}$ :

$$
\begin{gathered}
\beta_{3}=90-\alpha, \\
\alpha_{1}=\beta, \\
\alpha_{2}=\beta_{1}, \\
\alpha_{3}=\beta_{2} .
\end{gathered}
$$

По аналогии с первым случаем вычисляется $\left|A_{2} B_{2}\right|$. И также вычисляем $\left|A_{3} B_{3}\right|$ и $\left|A_{4} B_{4}\right|$.

$$
\begin{aligned}
& \left|A_{2} B_{2}\right|=D_{T} \operatorname{tg}\left(\beta_{1}\right), \\
& \left|A_{3} B_{3}\right|=d_{c} \operatorname{tg}\left(\beta_{2}\right), \\
& \left|A_{4} B_{4}\right|=L_{3} \operatorname{tg}\left(\beta_{3}\right) .
\end{aligned}
$$

Значения углов $\beta_{1}$ и $\beta_{2}==\beta$ определяются из следующих соотношений:

$$
\begin{aligned}
& \beta_{1}=\arcsin \left(\left(n_{c} \sin \alpha_{1}\right) / n_{l}\right), \\
& \beta_{2} \beta=\arcsin \left(\left(n_{a} \sin _{\alpha}\right) / n_{c}\right),
\end{aligned}
$$

где $n_{l}-$ показатель преломления текущей жидкости.

Значение $L_{2}$ определяется следующим соотношением:

$$
L_{2}=\left|A_{1} B_{1}\right|+\left|A_{2} B_{2}\right|+\left|A_{3} B_{3}\right|+\left|A_{4} B_{4}\right| .
$$

После подстановки в (14) соотношений (1)-(13) получаем следующее соотношение:

$$
\begin{aligned}
L_{2} & =d_{c} \operatorname{tg}\left(\arcsin \left(\left(n_{a} \sin \alpha\right) / n_{c}\right)\right) \\
& +D_{T} \operatorname{tg}\left(\arcsin \left(\left(n_{a} \sin \alpha\right) / n_{l}\right)\right) \\
& ++d_{c} \operatorname{tg}\left(\arcsin \left(\left(n_{a} \sin \alpha\right) / n_{c}\right)\right)+L_{3} \operatorname{ctg}(\alpha) .
\end{aligned}
$$

Анализ полученного выражения (15) показывает, что смещение максимума лазерного излучения $L_{2}$ по оси $O Y$ зависит как от показателя преломления текущей жидкости $n_{l}$, так и от угла $\alpha$ и расстояния $L_{3}$. Изменение последних двух параметров можно использовать в работе автоматической системы настройки оптической части проточного рефрактометра для обеспечения погрешности измерения $n_{l}$ менее $10^{-4}$ при изменении, например, температуры $T$ текущей жидкости.

Преобразуем (15) для получения соотношения для $n_{l}$ в явном виде:

$$
\begin{aligned}
& D_{T} \operatorname{tg}\left(\arcsin \left(\left(n_{a} \sin \alpha\right) / n_{l}\right)\right) \\
& =L_{2}-2 d_{c} \operatorname{tg}\left(\arcsin \left(\left(n_{a} \sin \alpha\right) / n_{c}\right)\right)-L_{3} \operatorname{ctg}(\alpha), \\
& n_{l}=\left(n_{a} \sin \alpha\right) / \sin \left(\operatorname { a r c t g } \left(\left(L_{2}\right.\right.\right. \\
& \left.\left.\left.-2 d_{c} \operatorname{tg}\left(\arcsin \left(\left(n_{a} \sin \alpha\right) / n_{c}\right)\right)-L_{3} \operatorname{ctg}(\alpha)\right) / D_{T}\right)\right) .
\end{aligned}
$$

Полученное соотношение (17) позволяет оценить, погрешности определения каких величин оказывают существенное влияние на погрешность $n_{l}$. Значение $n_{a}$ выбирается из градуировочных таблиц для измеренных значений давления, влажности и температуры у внешней стенки трубопровода. Поэтому погрешность na не оказывает существенного влияния на погрешность определения $n_{l}$.

Необходимо отметить, что изменение скорости течения жидкости в трубопроводе не оказывает влияние на определение значения $n_{l}$ с использованием (17) в отличие от других моделей проточных рефрактометров.

Для практической реализации данного метода измерения $n_{l}$ нами была разработана новая конструкция проточного рефрактометра. На рис. 2 представлена его структурная схема.

Лазерное излучение от полупроводникового лазера 1 поступает на оптическую систему 2 (призменный коллиматор), на выходе из которого преобразуется в узкую полосу размером $24 \mathrm{~mm}$ на $1 \mathrm{~mm}$. Далее лазерное излучение поступает на поворотную призму 3, которая используется для изменения его угла падения $\alpha$ на стенку трубопровода (рис. 2). Изменение угла $\alpha$ необходимо для измерения показателя преломления $n_{l}$ в широком диапазоне, так как при определенных значениях $\alpha$ и значениях $n_{l}$ может произойти явление ПВО на границе двух сред. Кроме того, температура текущей среды $T$ должна изменяться в широком диапазоне.

Призма 3 (рис. 2) в лабораторном макете рефрактометра размещается на поворотном оптическом столике (фирма STANDA, Литва), который располагается на оптической плите этой фирмы. Погрешность определения угла поворота в этом оборудовании составляет 0.1 ”. Поэтому погрешность определения $\alpha$ также не оказывает существенного влияния на погрешность определения $n_{l}$. В комплект оптической плиты входит оборудование для определения расстояния $L_{3}$. Дополнительно для определения расстояния $L_{3}$ нами использовался микроскоп, как в государственной поверочной схеме оптических измерителей расстояния. С учетом того, что необходимо обеспечить параллельное расположение стенки трубопровода 4 и фотодиодной линейки 6 в макете рефрактометра, погрешность измерения $L_{3}$ составляет значение порядка $0.04 \mathrm{~mm}$. В промышленном приборе, 


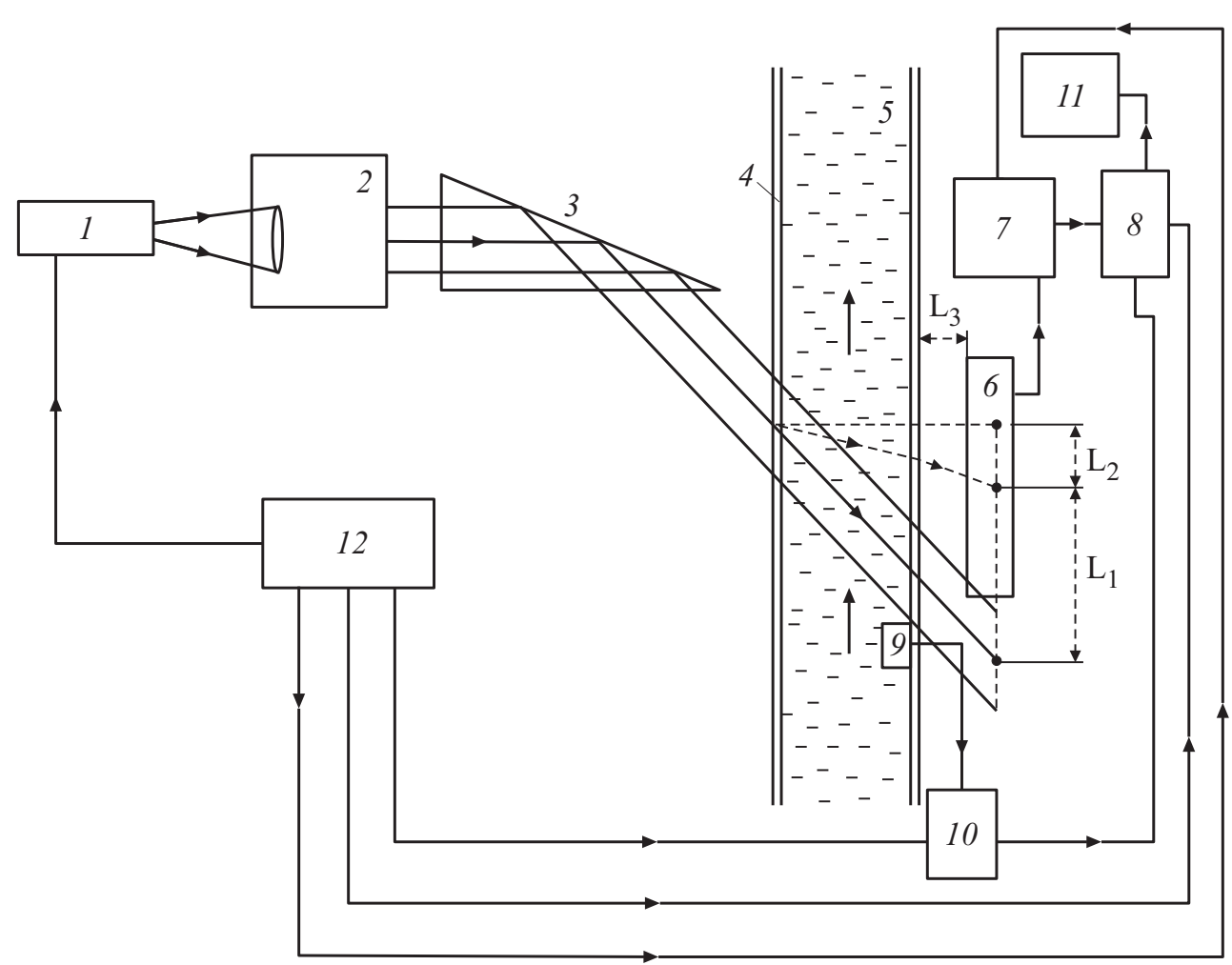

Рис. 2. Структурная схема лабораторного макета рефрактометра: 1 - полупроводниковый лазер; 2 - оптическая система; 3 поворотная (треугольная) призма; 4 - трубопровод; 5 - текущая жидкость; 6 - фотодиодная линейка; 7 - АЦП; 8 - устройство обработки и управления; 9 - датчик температуры; 10 - устройство для обработки информации с датчика температуры; $11-$ ноутбук; 12 -- многофункциональный источник питания.

например компании АТАГО, данная погрешность будет менее $0.015 \mathrm{~mm}$. И уже не будет оказывать существенного влияния на погрешность определения $n_{l}$.

В разработанной конструкции рефрактометра для измерения $n_{l}$ используется вставка в форме цилиндра из специализированного оргстекла ARCUMA 3M $\left(n_{c}=1.4953\right)$ с повышенной устойчивостью к нагреву с внешним диаметром $D_{T}=314 \mathrm{~mm}$. Толщина стенок оргстекла $d_{c}=2 \mathrm{~mm}$. Температура размягчения оргстекла ARCUMA 3M $T_{p}=569 \mathrm{~K}$. Стандартное оргстекло ARCUMA обладает $T_{p}=433 \mathrm{~K}$. Это является ограничением в использовании разработанной нами данной конструкции рефрактометра. Использование вставок из кварцевого стекла (температура размягчения более $1673 \mathrm{~K}$ ) требует дополнительных усложнений в конструкции рефрактометра, по причине хрупкости кварца по сравнению с оргстеклом, которые в ряде случаев реализовать крайне сложно.

Для исключения образования застойных зон и пустот при течении жидкости (малые значения расходов q) нами был предложен новый способ размещения разработанной вставки (на вертикальном участке трубопровода). Это значительно расширяет возможности использования разработанного метода в данной конструкции рефрактометра.
Использование вставок, состоящих из цилиндрического трубопровода с плоскими иллюминаторами $[17,20,23]$ в данной конструкции рефрактометра нецелесообразно по следующим причинам. При изменении $n_{l}$ в больших пределах, например при смене жидкости или изменении температуры, значение $L_{2}$ (смещение максимума лазерного излучения по оси $O Y$ относительно точки его падения $A$ на внешнюю стенку трубопровода) изменяется более чем на $10 \mathrm{~cm}$ (рис. 2). Это означает, что длина иллюминатора должна быть не менее $20 \mathrm{~cm}$. При быстрых потоках жидкости это приведет к образованию вихревых зон, в которых изменяется оптическая плотность текущей жидкости [24]. Изменение оптической плотности приведет к появлению дополнительной погрешности измерения показателя преломления $n_{l}[24,25]$. Кроме того, на стыках иллюминатора со стенками трубопровода могут образовываться инородные частицы, попадание которых в текущую жидкость может привести к непредсказуемым последствиям, особенно при контроле лекарственных препаратов, например корвалола.

При больших значениях диаметра трубопровода (в разработанном рефрактометре внутренний диаметр трубопровода составляет $310 \mathrm{~mm}$ ) и ширине полосы лазерного излучения $1 \mathrm{~mm}$ можно считать, что плоскость падения лазерного излучения на стенку трубопровода образует с ней угол, равный 90 градусов. В этом случае 


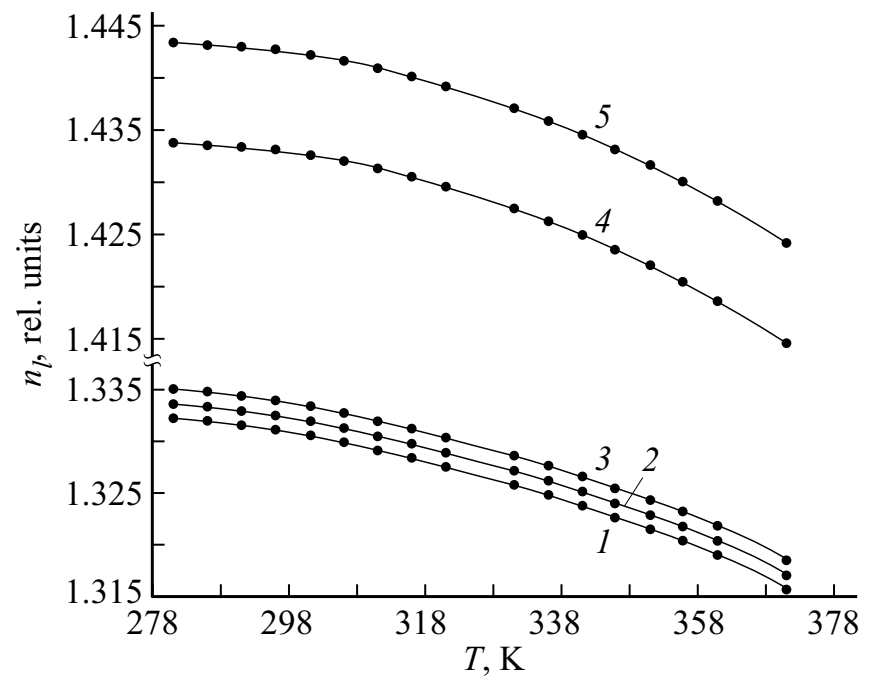

Рис. 3. Зависимость изменения показателя преломления $n_{l}$ жидких сред от температуры $T$. Графикам $1,2,3,4,5$ соответствуют следующие жидкие среды: дистиллированная вода, тяжелая вода, водопроводная вода, этиленгликоль, пропиленгликоль.

преломлением лазерного излучения на цилиндрических стенках трубопровода можно пренебречь (его влияние несущественно).

Для удобства обработки информации, поступающей с фотодиодной линейки 6 (рис. 2), после регистрации лазерного излучения осуществляется нормировка выходного сигнала $A$ по следующему принципу:

$$
A=\frac{\sum_{i=1}^{512} A_{i}}{A_{\max }},
$$

где $A_{\max }$ - максимальное значение сигнала освещенности с элемента фотодиодной линейки, $A_{i}$ - амплитуда сигнала с элемента фотодиодной ячейки, $i$ - номер элемента.

С фотодиодной линейки 6 сигнал через аналоговоцифровой преобразователь 7 поступает в устройство обработки информации и управления 8 , разработанное на основе микроконтроллера STM32 (ARM Cortex M3 core - STM32F100RBT6B). С микроконтроллера информация поступает на ноутбук 11, который используется для определения значения $L_{2}$. Далее вычисляется $n_{l}$. Информация о значении $\alpha$ и данные о среде, в которой находится трубопровод, предварительно заносятся в ноутбук.

Температура текущей жидкости в трубопроводе контролируется датчиками температуры 9. Информация от них через специальные устройства 10 поступает на устройство обработки информации 8 .

\section{2. Результаты экспериментальных исследований и их обсуждение}

Надежность и достоверность процесса измерения показателя преломления текущей жидкости $n_{l}$ с использованием разработанной нами конструкции проточного рефрактометра была проверена на нескольких жидких средах для различных температур $T$ (дистиллированная, тяжелая и водопроводная вода, а также этиленгликоль и пропиленгликоль). Эти среды постоянно используются в различных экспериментах, системах охлаждения и парогенераторах. При производстве этиленгликоля и пропиленгликоля, водные растворы которого в дальнейшем применяются против обледенения (например, самолетов, портовых буксиров, мостов и т.д.), используются быстрые потоки с высокой температурой. На рис. 3 представлены эти зависимости.

Показатели преломления этих сред также были измерены с помощью промышленного проточного рефрактометра PRM-100 alpha (ATAGO, Япония) - погрешность измерения $10^{-4}$. Полученные значения показателей преломления с использованием двух приборов совпали в пределах погрешности измерений. Кроме того, значения показателей преломления этих сред, измеренные на разработанном нами проточном рефрактометре, совпали со значениями показателей преломления, полученными ранее учеными с использованием других конструкций рефрактометров $[14,17,21,26,27]$. В ряде случаев жидкости при измерениях находились в стационарном состоянии, поэтому погрешность измерения показателя преломления была меньше, чем для текущей жидкости.

Кроме того, разработанный нами метод позволяет осуществить проверку достоверности значений $n_{l}$, полученных с применением рефрактометра, по „реперной“ метке (воздух). В трубопроводе вместо жидкости присутствует воздух $\left(n_{l}=n_{a}=1.0003\right)$. На границах двух сред (воздух-оргстекло) также происходит преломление лазерного излучения. Максимум лазерного излучения смещается по фотодиодной линейке 6 (рис. 2) на расстояние $L_{2}$. Это значение $L_{2}$ измеряется. Далее значение $L_{2}$ вычисляется с использованием (14) с учетом того, что $n_{l}=n_{a}$. Полученные результаты определения $L_{2}$ двумя способами совпали до третьего знака после запятой, что показывает высокую степень надежности и достоверности измерения $n_{l}$, полученных с использованием разработанного нами метода. Проверка осуществлялась несколько раз для различных значений углов $\alpha$ (рис. 2).

В проточных рефрактометрах в случае использования для измерения лазерного излучения прошедшего через текущую среду важным параметром является прозрачность среды. Большое влияние на погрешность измерения показателя преломления $n_{l}$ оказывает наличие в среде нерастворенных частиц различных размеров. В большинстве случаев их присутствие, а также высокая мутность среды являются ограничением в применении данного типа рефрактометра для контроля их состояния. 


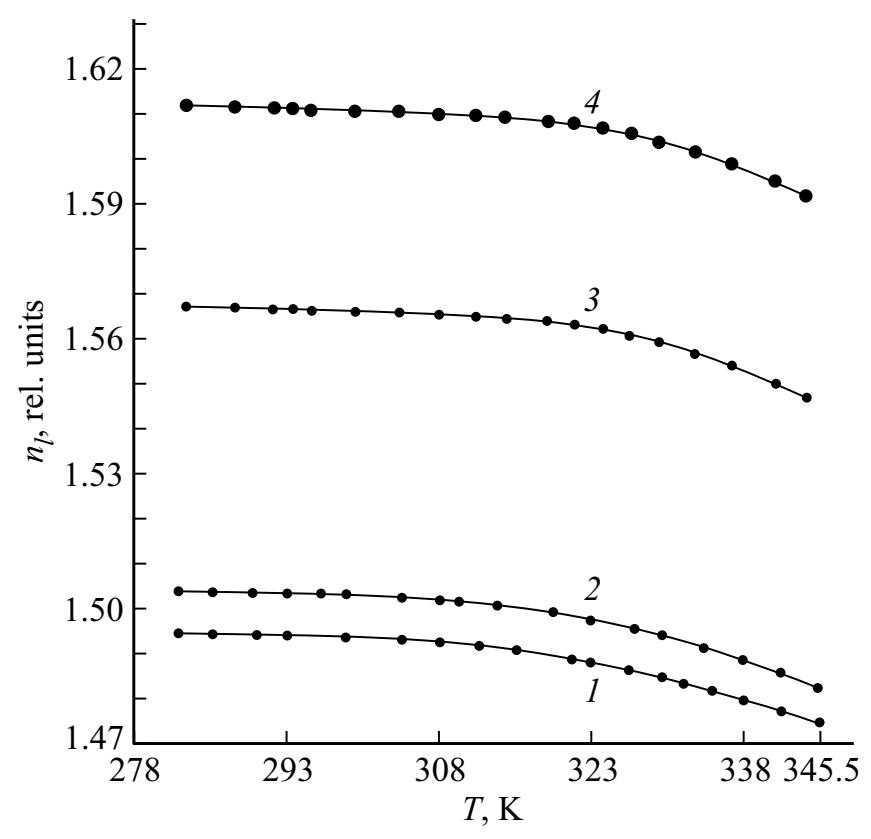

Рис. 4. Зависимость изменения показателя преломления $n_{l}$ различных сред, обладающих различной мутностью, от изменения температуры $T$. Графикам 1,2,3,4 соответствуют следующие жидкие среды: керосин, бензин Аи-95, моторное и трансформаторное масло.

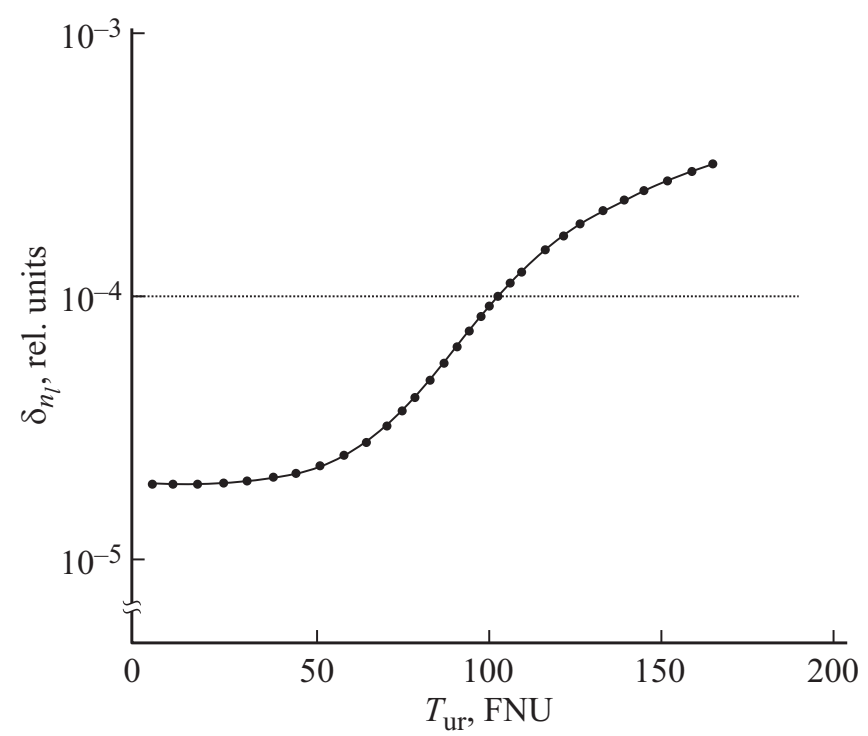

Рис. 5. Зависимость изменения погрешности измерения $\delta n_{l}$ текущей жидкости от её мутности $T_{u t}$ при температуре $T=293.3 \mathrm{~K}$.

На рис. 4 в качестве примера представлены зависимости изменения показателя преломления $n_{l}$ от температуры $T$ для различных сред (бензин, керосин, моторное и трансформаторное масло), обладающих различной мутностью.

Показатели преломления этих сред были также измерены с помощью промышленного проточного рефрактометра PRM-100 alpha - погрешность измерения $10^{-4}$.
Полученные значения показателей преломления бензина, керосина и моторного масла совпали в пределах погрешности измерений. Они также соответствуют данным, полученным другими учеными [22,26,28]. Для трансформаторного масла результаты измерения показателя преломления, полученные с использованием разработанной нами конструкции рефрактометра, различаются со значениями, полученными с использованием PRM-100 alpha. Кроме того, погрешность измерения показателя преломления трансформаторного масла в разработанной нами конструкции рефрактометра больше $10^{-4}$. Значение мутности трансформаторного масла составляет 284.17 FNU (мутность бензина составляет 7.85 FNU). Значение мутности всех исследуемых сред измерялось на стационарных пробах с использованием настольного ИК мутномера HI88713-02 (HANNA Instruments, США).

Для определения функциональных возможностей использования разработанного нами метода измерения показателя преломления $n_{l}$ в текущей жидкости нами были проведены исследования изменения погрешности измерения показателя преломления $\delta n_{l}$ текущей жидкости от её мутности $T_{u r}$. Для исследований использовалась смесь дистиллированной воды (мутность 0.73 FNU) и чернил (мутностью $168.62 \mathrm{FNU}$ ). Значение мутности исследуемой смеси изменялось с помощью изменения соотношения между концентрациями воды и чернил. На рис. 5 представлены результаты исследования изменения погрешности измерения ?nl текущей жидкости от её мутности $T_{u r}$.

Анализ проведенных исследований позволил установить предельное значение мутности среды (102.44 FNU), при котором значение погрешности измерения $\delta n_{l}$ в разработанном нами проточным рефрактометром не превышает $10^{-4}$. Дальнейшее увеличение мутности приводит к тому, что процессы поглощения лазерного излучения в текущей среде начинают уменьшать погрешность измерения $\delta n_{l}$ (мощность излучения лазера $l$ при измерениях $n_{l}$ оставалась неизменной).

\section{Заключение}

Полученные нами результаты исследований показали целесообразность использования разработанного нового метода измерения показателя преломления $n_{l}$ для контроля состояния текущей среды (с мутностью менее $102.44 \mathrm{FNU})$, особенно при турбулентном режиме её течения по трубопроводу.

Установлено, что погрешность измерения показателя преломления $\delta n_{l}$ текущей среды в разработанной конструкции проточного рефрактометра для реализации нового метода не зависит от скорости течения жидкости, что является большим преимуществом по сравнению с проточными рефрактометрами, принцип работы которых основан на явлении ПВО. 
Кроме того, проведенные эксперименты показали, что в отличие от методов измерения $n$, используемых в конструкциях проточных рефрактометров на основе явления ПВО, изменение температуры текущей среды $T$ в разработанном нами методе не оказывает существенного влияния на значение погрешности измерения $\delta n_{l}$.

Это позволяет в отличие других методов, применяемых в проточных рефрактометрах, особенно работающих на явлении ПВО, проводить измерения $n_{l}$ текущей жидкости в широком диапазоне её изменения, так как все ограничения по измерению $n_{l}$ в предложенной нами конструкции рефрактометра для реализации данного метода связаны с возможностями перемещением фотодиодной линейки по оси $O Y$, которое можно реализовать в пределах $20 \mathrm{~cm}$ вдоль оси трубопровода. Проведенные эксперименты показали, что этого достаточно для обеспечения измерения $n_{l}$ используемых в настоящее время жидкостей и их смесей с мутностью менее 102.44 FNU. Например, использование разработанного метода позволяет контролировать состояние текущего потока финилгидразина $n=1.6105$, который используется в медицинской промышленности при производстве лекарственных препаратов (например, антипирина или амидопирина). В настоящее время контроль состояния этого вещества в текущем потоке вызывает большие сложности.

\section{Список литературы}

[1] Кашаев Р.С., Газизов Э.Г. // ЖПС. 2010. Т. 77. № 3. C. $347-354$.

[2] Давыдов В.В. // Опт. и спектр. 2016. Т. 121. № 1. С. 18-25.

[3] Marusina M.Y., Bazarov B.A., Galaidin P.A., Marusin M.P., Silaev A.A., Zakemovskya E.Y., Mustaev Y.N. // Measurement Techniques. 2014. V. 57. N 5. P. 580-586.

[4] Даев Ж.А. // Измерительная техника. 2016. № 3. С. 29-31.

[5] Davydov V.V., Myazin N.S., Kiryukhin A.V. // Atomic Energy. 2020. V. 127. N 5. P. 274-279.

[6] Зубов В.А., Ринкевичюс Б.С. // Квант. электрон. 1997. Т. 24. № 12. C. $1161-1163$.

[7] Davydov R.V., Antonov V.I., Yushkova V.V. // J. Physics: Conference Series. 2019. V. 1236. N 1. P. 012079.

[8] D'yachenko S.V., Zhernovoi A.I. // Technical Physics. 2016. V. 61. N 12. P. $1835-1837$.

[9] Davydov V.V. // Measurement Techniques. 2017. V. 59. N 11. P. $1202-1209$.

[10] Marusina M.Y., Bazarov B.A., Galaidin P.A., Zakemovskaya E.Y., Mustafaev Y.N. // Measurement Techniques. 2014. V. 57. N 5. P. $580-586$.

[11] Вельт И.Д., Михайлова Ю.В. // Измерительная техника. 2013. № 3. C. 24-28.

[12] Chen J., Guo W., Xia M., Li W., Yang K. // Optics Express. 2018. V. 26. N 20. P. 25523.

[13] Grevtseva A.S., Smirnov K.J., Rud' V.Yu. // J. Physics: Conference Series. 2018. V. 1135(1). P. 012056.

[14] Шур В.Л., Найденов А.С., Лукин А.Я., Лейбенгард Г.И. // Измерительная техника. 2006. № 8. С. 50-53.

[15] Davydov V.V., Dudkin V.I., Karseev A.Yu., Vologdin V.A. // J. Appl. Spectrosc. 2015. V. 82. N 6. P. 1013-1019.
[16] Gabbasov B., Gafurov M., Starshova A., Mamin G., Orlinskii S. // J. Magnetism and Magnetic Materials. 2019. V. 470. P. $109-117$.

[17] Карабегов М.А. // Измерительная техника. 2004. № 4. C. 50-54.

[18] Davydov V.V., Kruzhalov S.V., Grebenikova N.M., Smirnov K.J. // Measurement Techniques. 2018. V. 61. N 4. P. $365-372$.

[19] Карабегов М.А. // Измерительная техника. 2007. № 6. C. $31-36$.

[20] Мищенко Ю.В. // Измерительная техника. 2007. № 12. C. $25-30$.

[21] Calhoun W.R., Maeta H., Combs A., Bali L.M., Bali S. // Opt. Lett. 2010. V. 35. N 8. P. 1224-1226.

[22] Calhoun W.R., Maeta H., Roy S., Bali L.M., Bali S. // J. Dairy Science. 2019. V. 93. N 8. P. 3497-3504.

[23] Karabegov M.A. // Measurement Techniques. 2011. V. 54. N 10. P. $996-1002$.

[24] Davydov V.V., Moroz A.V. // Opt. Spectrosc. 2020. V. 128. N 9. P. $1415-1420$.

[25] Карабегов М.А., Комарков Ю.И., Хуршудян С.А. // Измерительная техника. 1981. № 3. С. 64-66.

[26] Золотарев В.М., Морозов В.Е., Смирнова Е.В. Оптические постоянные природных и технических сред. СПб.: Лань, 2008. 348 с.

[27] Белов Н.П., Лапшов С.Н., Патяев А.С., Шерстобитова А.С., Яськов А.Д. // Научно-технический вестник информационных технологий, механики и оптики. 2012. T. 12. № 2. C. $138-139$.

[28] Karabegov M.A. // Measurement Techniques. 2012. V. 54. N 11. P. $1203-1212$. 